\title{
Serial position effects in social transmission of food preference: Retention/demonstration intervals
}

\author{
Benjamin Melchor-Hipólito, Gustavo Meza-Reynoso, C. Edith Juárez-Maldonado, \\ Guadalupe Ortega-Sanedra, Sara E. Cruz-Morales, and J. C. Pedro Arriaga-Ramírez \\ National Autonomous University of Mexico, Campus Iztacala, Tlalnepantla, Mexico
}

\begin{abstract}
The effects of retention and demonstration intervals on serial position were evaluated in two experiments with Long-Evans rats. A list of 3 demonstrators that had eaten one of three flavored foods was presented to naive observers. In Experiment 1, there were four groups, three groups with a retention interval compared with one group with a zero retention interval or no retention interval. In Experiment 2, the demonstration interval was reduced. Intervals of 15, 5, 2, and 1 min were used. In Experiment 1, primacy decreased gradually in the four groups as the retention interval was increased in duration. In Experiment 2, primacy also decreased gradually, and recency occurred with the 1-min demonstrator interval. The increase in the duration of the retention interval reduced primacy. The reduction in the duration of the demonstration interval decreased primacy and produced recency.
\end{abstract}

Lists of items are used to study memory in humans and in several animal species. In this procedure, a list of items is presented to the experimental subjects, and it has been found that the items at the beginning and end of the list are better remembered than items in the middle of the list. Taken together, these phenomena are called the $s e-$ rial position effect; the improved recall at the beginning and at the end of the list are termed primacy and recency, respectively.

Items in the middle of the list are not recalled as well as items at either end of the list (Atkinson \& Shiffrin, 1968). Primacy and recency effects have been found in several animal species (see Bolhuis \& van Kampen, 1988; Cook, Wright, \& Sands, 1991; Crystal \& Shettleworth, 1994; DiMattia \& Kesner, 1984; Harper, Dalrymple-Alford, \& McLean, 1992; Harper, McLean, \& Dalrymple-Alford, 1993; Reed, 2000a; Reed, Chih-Ta, Aggleton, \& Rawlins, 1991; Wright, 1994, 1998, 2002). Serial position effects have been found in humans, monkeys, and pigeons (see Santiago \& Wright, 1984; Wright, 1998, 2002; Wright, Santiago, Sands, Kendrick, \& Cook, 1985). With rats, primacy and recency have been found in spatial tasks (see Bolhuis \& van Kampen, 1988; DiMattia \& Kesner, 1984; Harper et al., 1992; Harper et al., 1993; Reed et al., 1991) and in nonspatial tasks, such as the flavor paradigm, in which the serial recall of flavored solutions is studied (see Reed, 2000a, 2000b; Reed, Croft, \& Yeomans, 1996). In those studies, rats consumed more of the first and last items of the lists presented following presentation of five novel, flavored solutions (Reed, 2000b). Reed (2000b) interpreted this difference in consumption as a serial position effect and concluded that the rela- tive novelty of the first flavor was not responsible for the effects observed. In that study, the main sources of information were the tastes and smells of the flavored liquids in the list.

There are several details in the methods of the studies mentioned above that should be noted. The radial-maze apparatus was used in the spatial-task studies with rats as subjects. In those studies, the subject was faced with a choice between two arms. In the flavor paradigm, the subject faced a choice of five flavored liquids. However, those methods differ from those that were used in studies with monkeys as subjects. In the studies with monkeys, the subject had to respond when it was faced with an item that had been presented in the list during training.

In adult rats, a rat that has eaten flavored food will influence a different, naive rat's preference for that food when it comes into contact with it (Galef \& Wigmore, 1983; Posadas-Andrews \& Roper, 1983), a phenomenon that illustrates the social transmission of food preferences and may be used to study memory processes. Eichenbaum (2002) used this paradigm as an example of social olfactory learning and memory when studying the involvement of the hippocampus in associative learning (Bunsey \& Eichenbaum, 1995). He wrote that "social transmission of food preferences provides a strong example of declarative-like (inferential) learning in the natural behavior of rats" (p. 136). Eichenbaum described this paradigm as a procedure in which learning involved stimulus-stimulus associations in a single training episode. In a social encounter without a feeding episode, the observer was exposed to an arbitrary stimulus that was reinforced by another natural stimulus, the demonstra-

J. C. P. Arriaga-Ramírez, jcpedro@servidor.unam.mx 
tor's breath. After the rats' learning, the memory test was done in a nonsocial situation that involved food-selection choices without the stimulus that reinforced learning about a new food odor. Finally, Eichenbaum mentioned that this aspect of the social transmission of food preference task, the expression of memory in a situation different from the learning trial, was strongly consistent with the declarative property of representational flexibility. Galef and Durlach (1993) found an absence of blocking (Kamin, 1969) in another study that evaluated the conditioned stimulus properties of the flavor of food that demonstrators had eaten. This absence of blocking demonstrated that rats were able to process at least two social stimuli in sequence without the first interfering with the second. This finding reported by Galef and Durlach is similar to that reported by Reed (2000a), in which the effects produced in the flavor paradigm were not produced by the relative novelty of the first flavor. One interesting issue then is whether rats are able to process olfactory cues in the same way they process visual and spatial cues, as has been shown in studies by Bolhuis and van Kampen (1988), DiMattia and Kesner (1984), Harper et al. (1992), and Harper et al. (1993). The studies relating to the flavor paradigm (Reed, 2000a, 2000b; Reed et al., 1996) suggest that the rats can process olfactory cues in this way.

Juárez-Maldonado et al. (2006) used the social transmission of food preference paradigm to test the recall of a list of demonstrators that had eaten one of three flavored foods. This paradigm has been used to study declarative memory in rats (Bunsey \& Eichenbaum, 1995). In Experiment 1 , in a list of 3 demonstrator rats that had eaten one of three different flavors of food, when an interstimulus interval (ISI) was observed between items, a recency effect was shown when this group was compared with a group in which this ISI was absent. In Experiment 2, Juárez-Maldonado et al. found that a von Restorff effect was produced when either the distinctiveness or context of the demonstrator presented in the middle of the list was changed - for example, when a rat of a different strain was placed as the second demonstrator. When the second demonstrator was presented in darkness, a primacy effect was shown when this group was compared with a control group in which the middle item was presented without changing either its distinctiveness or its context.

In addition to the ISI and the distinctiveness and context of the middle item, other parameters have been studied in serial recall- the effects of a retention interval after list termination, for example (Reed et al., 1996; Wright, 2002), and the duration of the item-viewing interval (Cook et al., 1991).

Reed et al. (1996) lengthened the retention interval in four experiments using the flavor paradigm $(0,6$, and $30 \mathrm{~min}$, and $24 \mathrm{~h}$ ). They found that, as the retention interval between training and testing was lengthened, the primacy produced by the shorter intervals also increased. With the longest interval, both primacy and recency were produced. Wright (2002) found that, when a rhesus monkey was tested with an auditory list followed by a retention interval $(0,1,2,10,20$, or $30 \mathrm{sec})$, there was a change from the primacy effect produced with the 0 -sec delay to a recency effect when the retention interval was lengthened. Wright (1998) mentioned that the serial-position functions of auditory lists were exactly the opposite of those produced with a visual list, and these were the same as the functions found with humans as subjects. When humans and monkeys were presented with a task that involved recalling a list of visual stimuli, a recency effect occurred with a 0 -sec delay, and primacy was increased as the delays were increased. One interesting question, then, is whether adding a retention interval after presenting a list of demonstrators to a naive observer would produce effects similar to those found with the flavor paradigm and with auditory lists with monkeys as subjects.

Cook et al. (1991) studied monkeys and found that, as the viewing time of the items in a list increased $(0.08$, $0.5,1,2$, and $4 \mathrm{sec})$, the accuracy also increased, and primacy was produced (A. A. Wright, personal communication, September 27, 2007). One interesting issue, then, is whether lengthening demonstration intervals of a list of demonstrators presented to naive observers would produce an effect similar to that produced when viewing time of items is increased.

Informed by the studies reviewed above, we designed the present study for the primary purpose of testing whether rats would show a retention interval effect after interacting with a list of 3 demonstrators, as was found by Reed et al. (1996) with rats and by Wright (2002) with monkeys. A second objective was to assess whether the effect of changing the duration of the interval in which demonstrators interacted with observers would change the effects produced by a long interval and, if so, whether a recency effect would be produced with a short interval, as was found by Cook et al. (1991) in their study with monkeys.

\section{EXPERIMENT 1}

In this experiment, a group of demonstrators that had eaten different flavored foods was presented to naive observers to determine whether a serial position effect would be produced without a retention interval. This group was compared with three other groups with different retention intervals after the interaction with the demonstrators had ended.

\section{Method}

Subjects. Forty-eight naive, female Long-Evans rats, 12 as demonstrators and 36 as observers, were used. The rats obtained from the university vivarium were 90 days old at the beginning of the experiment. Their free-feeding body weights ranged from 214 to $297 \mathrm{~g}$. The rats were food deprived, except when they had free access to chow (Harlan, Mexico City) for 20 min each day. The subjects were housed in individual steel boxes and had free access to water. The room where the rats were kept had a 12:12-h light:dark cycle and an ambient temperature between $21^{\circ}$ and $26^{\circ} \mathrm{C}$.

Apparatus and Materials. The subjects were housed individually in steel boxes (Armexa, Mexico City) that were $18 \mathrm{~cm}$ high, $18 \mathrm{~cm}$ wide, and $24 \mathrm{~cm}$ long. The demonstrators were fed in their home cages with powdered chow with flavor added. Food was mixed with one of three flavors: $1 \%$ cinnamon (McCormick, Mexico City), 
$2 \%$ cocoa (Hershey's, Mexico City), or $0.5 \%$ vanilla (Flor de Maria, Mexico City), all by weight. Demonstrations were performed in a Plexiglas box that was $15 \mathrm{~cm}$ high, $26 \mathrm{~cm}$ wide, and $36 \mathrm{~cm}$ long. For the preference test, flavored food was presented in metal containers that were $2.4 \mathrm{~cm}$ high, $5 \mathrm{~cm}$ wide, and $7.5 \mathrm{~cm}$ long. The containers were wired to the rear wall of a Plexiglas test box that was $20 \mathrm{~cm}$ high, $46 \mathrm{~cm}$ wide, and $53 \mathrm{~cm}$ long, and were separated by Plexiglas lids that were $1 \mathrm{~cm}$ high, $1 \mathrm{~cm}$ wide, and $15 \mathrm{~cm}$ long. The chamber and containers were cleaned after each observer had completed its 20 -min test. An Ohaus balance (Model 310) with a 0.01 -g resolution was used to weigh the amount of remaining food and to calculate the amount consumed by subjects.

Procedure. After 7 days of a feeding regimen of 20 -min access to food per day, four groups of 9 rats each were presented, sequentially, with 3 demonstrators that had each eaten, for $30 \mathrm{~min}$, one of three flavored foods. The powdered chow food was mixed with one of three flavors- $1 \%$ cinnamon, $2 \%$ cocoa, or $0.5 \%$ vanilla - before being given to the demonstrators. The demonstrators were also subjected to a feeding regimen so they would consume flavored food before the demonstration took place. There were 3 demonstrators for each group. The demonstrators always ate the same flavor of food before demonstrations. The order of demonstrator presentation was counterbalanced across observers. Each flavor of food was presented three times in each of the three positions in the list, both for demonstration and for testing. The lists used for demonstration and for testing were different. The naive observers interacted individually and for periods of $15 \mathrm{~min}$ with each of 3 demonstrators. The observers remained in the demonstration box, and the demonstrators were successively placed in the box for $15 \mathrm{~min}$. After demonstration, the observers were individually given a preference test in a Plexiglas test box in which three metal containers were wired to one side. The rats had access to $20 \mathrm{~g}$ of each of the three flavored foods, each placed in one of the three separate containers. The position of foods was counterbalanced across subjects. Preference was measured for $20 \mathrm{~min}$. The amount consumed was considered as much a measure of preference as an index of recall. For 9 of the subjects, "Group Zero," the demonstrators were presented one by one for periods of $15 \mathrm{~min}$ each. A preference test was initiated immediately after the last demonstrator had been presented for $15 \mathrm{~min}$. For the other three groups, a retention interval of 2,4 , or $24 \mathrm{~h}$ was observed after the demonstration had ended. During the retention intervals, the observer remained alone in its home cage. The duration of the retention interval was the only difference between the four groups.

\section{Results}

Figure 1 shows the mean percentage of flavored food consumed at each serial position for all of the groups in this experiment. An examination of the figure shows that a primacy effect was evident in Group Zero. In the other three groups, this effect was reduced gradually. A mixed ANOVA with group as a between-subjects factor and serial position as a within-subjects factor revealed a significant interaction of group and serial position $[F(6,64)=2.26$, $p=.05]$. A test of within-subjects contrasts revealed a significant linear trend for the interaction of serial position with group $[F(3,32)=2.87, p=.05]$. Trend tests were conducted first on combined groups and subsequently on individual groups, using an ANOVA test with group and serial position as factors. This test revealed a significant effect of group $[F(3,96)=4.29, p=.01]$, an unreliable effect of serial position $(F<1)$, and a significant interaction between group and serial position $[F(6,96)=3.39$, $p<.01]$. A simple contrast using Group Zero as the reference category for comparisons with each of the other three groups was significant $[F(1,96)=7.28, p=.01]$. Planned comparisons showed a nonsignificant global trend for serial position $(F<1)$. Individual planned comparisons for trend showed a significant linear trend for Group Zero $[F(1,96)=7.99, p<.01]$ and a significant quadratic trend for the 24-h group $[F(1,96)=6.09, p=.02]$.

This trend analysis is appropriate when the variancecovariance matrix of the experiment has compound symmetry. When this condition is met, the constants associ-

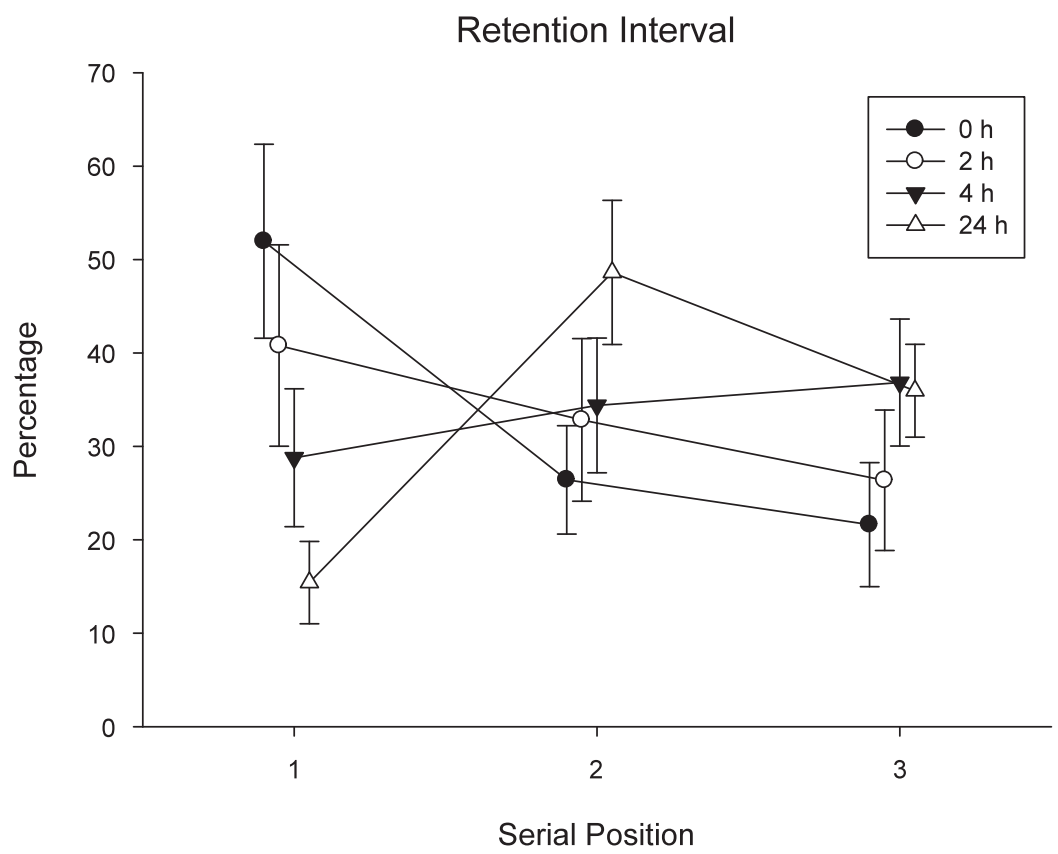

Figure 1. Mean percentage of flavored food consumed in each of the serial positions at different retention intervals in Experiment 1. Bars show standard errors of the means. 
ated with each subject that form the covariance between the repeated measures and the experimental errors are independent (Winer, 1971). The critical value for a .05 test of the hypothesis that the compound symmetry condition is met is $\chi_{95}^{2}(4)=9.5$. Since the value obtained for this test is 4.37 , the data do not contradict the hypothesis.

Repeated measures ANOVAs on the percentage of flavored food consumed (grouped by flavor) did not show a reliable difference in the amount of individual flavors consumed $(p>.05)$.

\section{Discussion}

The primacy effect seen in Group Zero decreased in an orderly fashion, and the simple contrast showed that this difference was reliable. Also, the significant trends of Group Zero and the 24-h groups add to the reliability of this change. These results are different from those obtained by Reed et al. (1996) with the flavor paradigm. Reed et al. (1996) found that an increase in primacy and recency was produced with the longest retention interval. However, the results of the present experiment are similar to those obtained by Wright (2002) on retention of auditory lists by monkeys. Wright found that, as the retention interval was lengthened, primacy was reduced, and a recency effect was produced; however, in the present experiment, primacy was reduced as the interval was lengthened, but no recency was produced.

\section{EXPERIMENT 2}

In studies of list recall, it has been found that, as the viewing time of items was increased, accuracy also increased, and primacy was produced (Cook et al., 1991).
The purpose of this experiment was to determine whether increasing the time that observers interacted with demonstrators would produce an effect similar to that produced by increasing the viewing time of items.

\section{Method}

Subjects. Sixty naive, female Long-Evans rats were used. From these rats, 12 were used as demonstrators, and 48 were used as observers. The ages of the subjects were the same as in Experiment 1, and the free-feeding body weight range was 200 to $289 \mathrm{~g}$.

Apparatus and Materials. The apparatus and materials were identical to those used in Experiment 1.

Procedure. After 7 days of a feeding regimen of 20-min access to food per day, a list of 3 demonstrators, each having eaten a different flavored food, was presented successively to each of 12 observers in each of the four groups. The powdered chow food was mixed with one of three flavors - $1 \%$ cinnamon, $2 \%$ cocoa, or $0.5 \%$ vanillabefore being given to the demonstrators. The demonstrators were also subjected to a feeding regimen, so that they would consume flavored food before the demonstration took place. This was done in the demonstration box. There were three demonstrators for each group. Demonstrators ate the same flavor of food before demonstrations. According to the demonstration intervals, subjects were assigned to one of the following groups: 1, 2, 5, or $15 \mathrm{~min}$. Immediately after each demonstration ended, the observers were given a 20 -min preference test, in which they had access to $20 \mathrm{~g}$ of each of the flavored foods - cinnamon, cocoa, and vanilla - with positions balanced in the test box. The lists for demonstration and for testing were different. Each flavor was presented four times in each of the three positions on the list for both demonstration and testing.

\section{Results}

Figure 2 presents the mean percentage of flavored food consumed at each position for all of the groups in this experiment. In the 1-min group, a recency effect was produced, in contrast to a primacy effect in the 15 -min group.

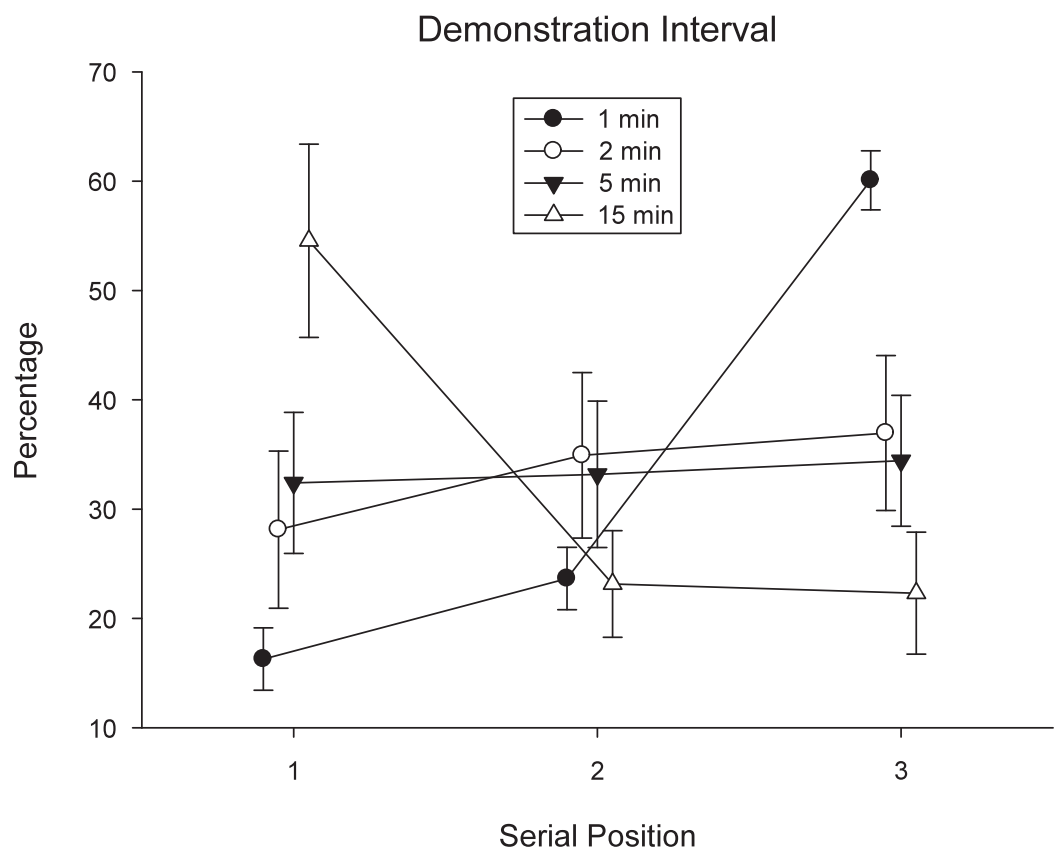

Figure 2. Mean percentage of flavored food consumed in each of the serial positions at different demonstration intervals in Experiment 2. Bars show standard errors of the means. 
In the 2- and 5-min groups, this primacy effect was not observed; all three items were recalled about the same. A repeated measures ANOVA with group as a betweengroups factor and serial position as a within-subjects factor revealed a nonsignificant effect of position $[F(2,88)=$ $1.74, p=.18]$, a significant effect of group $[F(3,44)=$ $3.56, p=.022]$, and a significant interaction between serial position and group $[F(6,88)=4.96, p<.001]$. A within-subjects contrast revealed a significant linear trend for the interaction of serial position with group $[F(3,44)=$ $8.20, p=.001]$.

Trend tests were conducted first on combined groups and later on individual groups, using an ANOVA test with group and serial position as factors. This test revealed a significant effect of group $[F(3,132)=7.01, p=.0002]$, a significant effect of serial position $[F(2,132)=15.06$, $p<.0001]$, and a significant interaction between group and serial position $[F(6,132)=7.44, p<.0001]$. A simple contrast using the 15-min group as the reference category for comparison with each of the other three groups was significant $[F(1,132)=17.21, p<.001]$. Planned comparisons revealed a significant global linear trend for serial position $[F(1,132)=26.27, p<.001]$. In addition, this test revealed a significant interaction between group and serial position $[F(6,132)=7.44, p<.0001]$. Planned comparisons for individual groups revealed a significant linear trend for the 15-min group $[F(1,132)=14.23, p<$ $.001]$ and the 1 -min group $[F(1,132)=26.27, p<.001]$.

This trend analysis is possible when the variancecovariance matrix of the experiment has compound symmetry. When this condition is met, the constants associated with each subject that form the covariance between the repeated measures and the experimental errors are independent (Winer, 1971). The critical value for a .05 test of the hypothesis that the compound symmetry condition is met is $\chi_{.95}^{2}(4)=9.5$. Since the value obtained for this test is 2.28 , the data do not contradict the hypothesis.

A repeated measures ANOVA on the percentage of flavored food consumed (grouped by flavor) did not show a reliable difference in the amount of individual flavors consumed $(p>.05)$.

\section{Discussion}

In this experiment, the smallest demonstration time in the 1-min group produced a recency effect. This recency effect was eliminated when demonstration time was changed in the other three groups to 2, 5, and $15 \mathrm{~min}$. In addition, a primacy effect was observed in the 15-min group. This result is similar to that obtained in the study of Cook et al. (1991), who found that increasing viewing time increased both accuracy and primacy. This effect is not shown in the Cook et al. article, but it was confirmed by Wright (A. A. Wright, personal communication, September 27, 2007).

\section{GENERAL DISCUSSION}

One question asked in these experiments was whether adding a retention interval at the end of a list of demon- strators would produce an effect similar to those found in previous experiments. Experiment 1 showed that the primacy effect observed in Group Zero was reduced as the retention interval was lengthened. In studies with monkeys recalling an auditory list (Wright, 2002), primacy effects have been found to decrease as the retention interval is lengthened. These results are different from those obtained by Reed et al. (1996) with the flavor paradigm. Reed et al. (1996) found that increases in primacy and recency were produced with the longest retention interval. There were procedural differences between the present study and the Reed et al. (1996) experiment, in which the list items were flavored liquids and the lists were formed with five items. In the present experiment, list items were odor and social stimuli presented by demonstrators, and the lists were formed with three items. These differences may explain why different results were obtained in these studies, in which lists of items were recalled.

The procedures that have shown primacy effects in animals have been questioned for various reasons, including the following. (1) The variance observed was lower than expected on the basis of the binomial model; (2) the data were selected on the basis of response criteria; and (3) explicit observing responses to the first item in the list were required of subjects (Gaffan, 1992; Gaffan \& Gaffan, 1992). Reed $(1992,1994)$ mentioned that the variance predicted by the binomial model may not be appropriate for these types of studies. Wright (1994) also noted that primacy effects are not produced by list-initiation responses. In the present study, the subjects that showed primacy in Experiment 1 and those that showed primacy in Experiment 2 were not selected by any criteria, no observing response was required of them, and their responses were not classified as "correct" or "incorrect."

Another question asked in Experiment 2 was whether changing the duration of the demonstration intervals would produce effects similar to those observed in monkeys, with which viewing time of items was increased. The results of Experiment 2 show that the primacy effect seen in the 15-min group decreased as the duration of the demonstration intervals was reduced in the other groups. The reduction in interval duration may be similar to the reduction of viewing time used in the experiments with monkeys (Cook et al., 1991). The effects seen in the 2- and 5 -min groups may represent a transitional effect between all recency or all primacy, dependent on certain values of the parameters manipulated in list elaboration, as Wright (1994) mentioned. In addition, the increase in recency seen in the 1-min group is similar to the effect found by Cook et al. with viewing time.

Cook et al. (1991) found that, as the viewing time of items increased, the accuracy also increased, and primacy was produced. This effect could be similar to a rehearsal effect found in humans (Wright et al., 1990), but several studies have not shown enough evidence to conclude that monkeys may rehearse (Wright, 1998). Wright (1998) added that a passive memory process may be responsible for the primacy effect seen in animals. Primacy effects seen at long viewing times with monkeys and long 
demonstration intervals with rats may involve a passive memory process.

As Wright (1998) mentioned, the fact that a passive memory process is involved in a dynamic system that produces both primacy and recency raises doubts about the modal model and its limited capacity for short-term storage (Atkinson \& Shiffrin, 1968) in analyzing animal serial position effects.

The serial-recall procedure used in the experiments reported here may seem similar to other ordered-stimuli relations. Some studies required trained subjects to respond to the sequential order of stimuli (D'Amato \& Colombo, 1988; Dusek \& Eichenbaum, 1997; Terrace, 1991). However, in those studies, extensive training was given to assess whether subjects would learn item series. The effects found in those studies were associative transitivity, chunking, or transitive inference. In the social transmission of preference paradigm used here, observers were given a single trial with each demonstrator. The procedure used in the experiments presented here was similar to those used in the spatial tasks in radial mazes (Bolhuis \& van Kampen, 1988; DiMattia \& Kesner, 1984; Harper et al., 1992; Harper et al., 1993; Reed et al., 1991) and to that used in the flavor paradigm (Reed, 2000a, 2000b; Reed et al., 1996). In those studies, after training, subjects were faced with choices between two arms of a maze or with five flavored solutions. In our procedure, observers faced a choice of three flavored foods, with position counterbalanced for demonstration and testing.

The effects reported here may be interpreted as results of blocking (Kamin, 1969). However, if that were the case, primacy would have been produced in all groups, because the first flavor would have blocked the recall of the other two flavors. The results of both experiments showed that manipulation of retention interval and of demonstration time reduced the primacy effect produced with the 0 - and 15-min values. A recency effect was even produced with the 1-min demonstrator interval. This reduction in primacy and the recency effect show that blocking was not responsible for the serial effects produced here.

\section{AU'THOR NOTE}

This research was supported by PAPIIT UNAM IN301106 and PAPCA (2003). The authors thank M. Fernanda Mata-Reza and Addalid Sánchez-Hernández for assistance in running Experiment 2. Correspondence concerning this article should be addressed to J. C. Pedro Arriaga-Ramírez, UNAM Campus Iztacala, División de Investigación y Posgrado, UIICSE $2^{\circ}$ Piso Cub. 15, Av. De los Barrios \#1, Los Reyes Iztacala, Tlalnepantla, Edo. Mexico AP 314, CP 54090, Mexico (e-mail: jcpedro@servidor.unam.mx).

\section{REFERENCES}

AtKinson, R. C., \& Shiffrin, R. M. (1968). Human memory: A proposed system and its control processes. In K. W. Spence \& J. T. Spence (Eds.), The psychology of learning and motivation: Advances in research and theory (Vol. 2, pp. 89-195). New York: Academic Press.

Bolhuis, J. J., \& VAN KAMPEN, H. S. (1988). Serial position curves in spatial memory of rats: Primacy and recency effects. Quarterly Journal of Experimental Psychology, 40B, 135-149.

Bunsey, M., \& Eichenbaum, H. (1995). Selective damage to the hippocampal region blocks long-term retention of a natural and non- spatial stimulus-stimulus association. Hippocampus, 5, 546-556. doi:10.1002/hipo.450050606

CoOK, R. G., Wright, A. A., \& SANDS, S. F. (1991). Interstimulus interval and viewing time effects in monkey list memory. Animal Learning \& Behavior, 19, 153-163.

Crystal, J. D., \& Shettleworth, S. J. (1994). Spatial list learning in black-capped chickadees. Animal Learning \& Behavior, 22, 77-83.

D'Aмато, M. R., \& Cоцомво, M. (1988). Representation of serial order in monkeys (Cebus apella). Journal of Experimental Psychology: Animal Behavior Processes, 14, 131-139. doi:10.1037/0097 $-7403.14 .2 .131$

DiMattia, B. V., \& Kesner, R. P. (1984). Serial position curves in rats: Automatic versus effortful information processing. Journal of Experimental Psychology: Animal Behavior Processes, 10, 557-563. doi:10.1037/0097-7403.10.4.557

Dusek, J. A., \& Eichenbaum, H. (1997). The hippocampus and memory for orderly stimulus relations. Proceedings of the National Academy of Sciences, 94, 7109-7114.

Eichenbaum, H. (2002). The cognitive neuroscience of memory: An introduction. New York: Oxford University Press.

Gaffan, E. A. (1992). Primacy, recency, and the variability of data in studies of animals' working memory. Animal Learning \& Behavior, 20, 240-252.

GAFFAN, E. A., \& GAFFAN, D. (1992). Less-than-expected variability in evidence for primacy and von Restorff effects in rats' nonspatial memory. Journal of Experimental Psychology: Animal Behavior Processes, 18, 298-301. doi:10.1037/0097-7403.18.3.298

GaleF, B. G., JR., \& Durlach, P. J. (1993). Absence of blocking, overshadowing, and latent inhibition in social enhancement of food preferences. Animal Learning \& Behavior, 21, 214-220.

GALEF, B. G., JR., \& WigmORE, S. W. (1983). Transfer of information concerning distant foods: A laboratory investigation of the "informationcentre" hypothesis. Animal Behaviour, 31, 748-758. doi:10.1016/ S0003-3472(83)80232-2

Harper, D. N., Dalrymple-Alford, J. C., \& Mclean, A. P. (1992). Production of a serial position effect in rats using a 12 -arm radial maze. Journal of Neuroscience Methods, 44, 197-207.

Harper, D. N., Mclean, A. P., \& Dalrymple-Alford, J. C. (1993). List item memory in rats: Effects of delay and delay task. Journal of Experimental Psychology: Animal Behavior Processes, 19, 307-316. doi:10.1037/0097-7403.19.4.307

Juárez-Maldonado, C. E., Ortega-Saavedra, G., HuichanOlivares, F., Gómez-Romero, J., Cruz-Morales, S. E., \& ArriagaRAMíreZ, J. C. P. (2006). Serial position effects in social transmission of food preference. Learning \& Behavior, 34, 374-378.

Kamin, L. J. (1969). Predictability, surprise, attention, and conditioning. In B. A. Campbell \& R. M. Church (Eds.), Punishment and aversive behavior (pp. 279-296). New York: Appleton-Century-Crofts.

Posadas-Andrews, A., \& Roper, T. J. (1983). Social transmission of food-preferences in adult rats. Animal Behaviour, 31, 265-271. doi:10.1016/s0003-3472(83)80196-1

ReED, P. (1992). Fewer doubts concerning rats' serial position performance: Reply to Gaffan and Gaffan (1992) and Rawlins, Deacon, Chih-Ta, and Aggleton (1992). Journal of Experimental Psychology: Animal Behavior Processes, 18, 305-307. doi:10.1037/0097 $-7403.18 .3 .305$

REED, P. (1994). Less than expected variance in studies of serial position effects is not sufficient reason for caution. Animal Learning \& Behavior, 22, 224-230.

ReED, P. (2000a). Rats' memory for serially presented flavors: Effects of interstimulus interval and generalization decrement. Animal Learning \& Behavior, 28, 136-146.

ReED, P. (2000b). Relative novelty does not explain primacy effects in rats' memory for serially presented novel flavors. Learning \& Motivation, 31, 99-113.

Reed, P., Chin-Ta, T., Aggleton, J. P., \& Rawlins, J. N. P. (1991). Primacy, recency, and the von Restorff effect in rats' nonspatial recognition memory. Journal of Experimental Psychology: Animal Behavior Processes, 17, 36-44. doi:10.1037/0097-7403.17.1.36

Reed, P., Croft, H., \& Yeomans, M. (1996). Rats' memory for serially presented novel flavours: Evidence for non-spatial primacy effects. Quarterly Journal of Experimental Psychology, 49B, 174-187. 
Santiago, H. C., \& Wright, A. A. (1984). Pigeon memory: Same/ different concept learning, serial probe recognition acquisition, and probe delay effects on the serial-position function. Journal of Experimental Psychology: Animal Behavior Processes, 10, 498-512. doi:10.1037/0097-7403.10.4.498

Terrace, H. S. (1991). Chunking during serial learning by a pigeon: I. Basic evidence. Journal of Experimental Psychology: Animal Behavior Processes, 17, 81-93. doi:10.1037/0097-7403.17.1.81

Winer, B. J. (1971). Statistical principles in experimental design (2nd ed.). New York: McGraw-Hill.

Wright, A. A. (1994). Primacy effects in animal memory and human nonverbal memory. Animal Learning \& Behavior, 22, 219-223.

Wright, A. A. (1998). Auditory and visual serial position functions obey different laws. Psychonomic Bulletin \& Review, 5, 564-584.
Wright, A. A. (2002). Monkey auditory list memory: Tests with mixed and blocked retention delays. Animal Learning \& Behavior, 30, 158164.

Wright, A. A., Cook, R. G., Rivera, J. J., Shyan, M. R., Neiworth, J. J., \& Jitsumori, M. (1990). Naming, rehearsal, and interstimulus interval effects in memory processing. Journal of Experimental Psychology: Learning, Memory, \& Cognition, 16, 1043-1059. doi:10.1037/0278-7393.16.6.1043

Wright, A. A., Santiago, H. C., Sands, S. F., Kendrick, D. F., \& Cook, R. G. (1985). Memory processing of serial lists by pigeons, monkeys, and people. Science, 229, 287-289.

(Manuscript received July 29, 2009;

revision accepted for publication September 1, 2009.) 